\title{
FACTORES ASOCIADOS A DEPRESIÓN EN PACIENTES CON CÁNCER DE MAMA
}

\author{
FACTORS ASSOCIATED WITH DEPRESSION IN PATIENTS WITH BREAST CANCER
}

Ximena Arbulú-La Torre ${ }^{1}$

\begin{abstract}
RESUMEN
Objetivo: Sistematizar los hallazgos de los estudios sobre los factores asociados a depresión en pacientes con cáncer de mama. Métodos: Se realizó una revisión sistemática de la literatura médica en Medline, SciELO y Google Scholar con los términos descriptores del Medical Subject Headings: depression, breast cancer. Se seleccionaron 6 artículos con los criterios de exclusión de artículos con un periodo de publicación entre 2013-2018, escritos en español o inglés, que analizaban a pacientes con depresión asociado a cáncer de mama y estudios con una muestra mínima de 80 participantes. Resultados: En esta revisión se han incluido 6 estudios. Los factores asociados a depresión en pacientes con cáncer de mama tenemos está la edad donde se ve que la población entre 40 y 60 años, estar divorciada o soltera, grado de instrucción hasta primaria, estar desempleado, pertenecer a una clase social baja, el estadío clínico 4 y el tratamiento de quimioterapia aumenta el riesgo de tener depresión en pacientes con cáncer de mama. Conclusión: Se concluye en la presente revisión que hace falta más estudios a mayor escala respecto a los factores asociados a depresión en pacientes con cáncer de mama especialmente a nivel de Perú, ya que se encontró solo un artículo relacionado al tema de interés. Por otra parte hace falta sistematizar bien los factores asociados ya que se encuentran diversos factores relacionados a depresión y con diferentes resultados dependiendo del tipo de hospital y de país, para poder plantear un buen programa de apoyo psicológico y educación sobre la enfermedad.
\end{abstract}

Palabras clave: Depresión; Cáncer ; Cáncer de mama; Revisión sistematica. (fuente: DeCS BIREME)

\begin{abstract}
Objective: Systematize the findings of the studies on the factors associated with depression in patients with breast cancer. Methods: A systematic review of the medical literature was made in Medline, SciELO and Google Scholar with the descriptive terms of the Medical Subject Headings: depression, breast cancer. Six articles were selected with the exclusion criteria of articles with a publication period between 2013-2018, written in Spanish or English, which analyzed patients with depression associated with breast cancer and studies with a minimum sample of 80 participants. Results: In this review, 6 studies have been included. The factors associated with depression in patients with breast cancer we have this age where it is seen that the population between 40 and 60 years, be divorced or single, education grade up to primary, be unemployed, belong to a low social class, the stadium clinical trial 4 and chemotherapy treatment increases the risk of depression in patients with breast cancer. Conclusion: It is concluded in the present review that more studies on a larger scale are needed regarding the factors associated with depression in patients with breast cancer, especially the level of Peru, since only one article related to the topic of interest was found. On the other hand, it is necessary to systematize the associated factors well since there are several factors related to depression and with different results depending on the type of hospital and country, in order to propose a good program of psychological support and education about the disease.
\end{abstract}

Key words: Depression; Cancer; Breast cancer; Systematic review. (source: MeSH NLM)

'Alumna de 10mo ciclo- Universidad Ricardo Palma, Lima-Perú.

Citar como: Ximena Arbulú-La Torre. Factores asociados a depresión en pacientes con cáncer de mama. [Artículo de Revisión].2019;19(1):74-79. (Enero 2019). DOI 10.25176/RFMH.v19.n1.1795

Journal home page: http://revistas.urp.edu.pe/index.php/RFMH

(c) Los autores. Este artículo es publicado por la Revista de la Facultad de Medicina Humana, Universidad Ricardo Palma. Este es un artículo de Open Access distribuido bajo los términos de la Licencia Creative Commons Atribución-NoComercial-Compartirlgual 4.0 Internacional.(http://creativecommons.org/licenses/by-nc-sa/4.0/), que permite el uso no comercial, distribución y reproducción en cualquier medio, siempre que la obra original sea debidamente citadas. Para uso comercial, por favor póngase en contacto con revista.medicina@urp.pe 


\section{INTRODUCCIÓN}

Mundialmente el cáncer de mama es la enfermedad oncológica más frecuente en mujeres, representando el $25,2 \%$ de todas las neoplasias y encabeza las causas de mortalidad por cáncer (14,7\%).

En el Perú esta neoplasia es la segunda más frecuente en mujeres y es la tercera causa de mortalidad por cáncer $(8,7 \%)$. Teniendo solamente en la costa peruana un $83 \%$ de las defunciones en mujeres; y dentro de esta Lima cuenta con el mayor número de mujeres con este padecimiento ${ }^{7}$.

El cáncer de mama al ser una enfermedad crónica afecta el bienestar emocional y el ajuste psicológico de las pacientes que lo padecen. El simple diagnóstico de cáncer supone una situación estresante y el posterior desarrollo de la enfermedad afecta diversos ámbitos de la vida, como el personal, laboral, académico o social, lo que implica un considerable reajuste físico y psicológico ya que, además de tener que enfrentarse a la enfermedad y sus tratamientos, las mujeres deben adaptarse a los cambios en su imagen corporal y a los sentimientos y actitudes que este con lleva ${ }^{4}$.

Por lo que el diagnóstico de cáncer con frecuencia se relaciona con comorbilidades psiquiátricas como depresión y ansiedad, complicando la recuperación de la intervención quirúrgica, aumentando el riesgo de la no adherencia a los tratamientos y al aislamiento, generando mayor sintomatología y sufrimiento en estas pacientes ${ }^{7}$.

Considerando la importancia de los síntomas emocionales en los pacientes con cáncer y el impacto de esta. Se plantea el presente artículo con el propósito de evaluar los factores asociados a depresión en pacientes con cáncer de mama.

\section{MÉTODOS}

Se realizó una revisión sistemática de la literatura médica acerca de los los factores asociados a depresión en pacientes con cáncer de mama. Para tal propósito se seleccionaron y revisaron los artículos científicos publicados desde enero de 2012 hasta el 2018, en los idiomas de español e inglés.

La búsqueda búsqueda bibliográfica se efectuó en la bases de datos electrónicas Medline, SciELO y Google Scholar con los términos descriptores del Medical Subject Headings: depression, breast cáncer.

Los criterios de selección fueron:

- Estudios que analicen la depresión en pacientes con cáncer de mama en función de múltiples variables.

- Artículos con un periodo de publicación entre 20132018, escritos en español o inglés.

- Artículos que analizaban a pacientes con depresión asociado a cáncer de mama.

- Estudios con una muestra mínima de 80 participantes.

A partir del conjunto de artículos que describían los factores asociados a depresión en pacientes con cáncer de mama, se agruparon los datos en tablas tomando en cuenta el título, el autor o autores, el año, el país, la muestra y el tipo de estudio (Tabla 1).

En una segunda fase se agruparon en una segunda tabla las variables relacionadas a los factores asociados a depresión en pacientes con cáncer de mama: Edad, grado de instrucción, trabajo, convivencia, tipo de cirugía y tipo de tratamiento.

Por último en la tabla tres se agrupo los factores asociados al tratamiento y estadío clínico en pacientes con cáncer de mama.

\section{RESULTADOS}

Tabla 1. Principales características de los estudios incluidos en la revisión.

\begin{tabular}{|c|c|c|c|c|c|}
\hline TÍTULO & AUTOR & AÑo & $\begin{array}{l}\text { PAÍS/ } \\
\text { CONTINENTE }\end{array}$ & MUESTRA & $\begin{array}{l}\text { TIPO DE } \\
\text { ESTUDIO }\end{array}$ \\
\hline $\begin{array}{l}\text { Trastornos de ansiedad y depresión en relación con la } \\
\text { calidad de vida de pacientes con cáncer de mama en } \\
\text { estadio localmente avanzado diseminado }\end{array}$ & $\begin{array}{l}\text { Martha Carolina } \\
\text { et al. }\end{array}$ & 207 & Colombia & 107 & Cohorte \\
\hline $\begin{array}{l}\text { Anxiety and depression in women with breast cancer: } \\
\text { Social and clinical determinants and influence of the } \\
\text { social network and social support (DAMA cohort) }\end{array}$ & $\begin{array}{l}\text { R. Puigpinós-Riera } \\
\text { et al. }\end{array}$ & 2018 & España & 1086 & Cohorte \\
\hline Depression in breast cancer patients & $\begin{array}{l}\text { Jovana Cvetkovic } \\
\text { et al. }\end{array}$ & 2017 & Irlanda & 87 & Cohorte \\
\hline $\begin{array}{l}\text { Prevalencia y factores asociados a la sintomatología } \\
\text { depresiva en mujeres con cáncer de mama en un hospital } \\
\text { público de Lima, Perú }\end{array}$ & $\begin{array}{l}\text { Silvia Berrospi- } \\
\text { Reyna et al. }\end{array}$ & 2017 & Perú & 385 & $\begin{array}{l}\text { Transversal } \\
\text { Analítico }\end{array}$ \\
\hline $\begin{array}{l}\text { Anxiety and Depression among Breast Cancer Patients in } \\
\text { an Urban Setting in Malaysia }\end{array}$ & $\begin{array}{l}\text { Mohd Rohaizat } \\
\text { Hassan et al. }\end{array}$ & 2015 & Malasya & 205 & Transversal \\
\hline $\begin{array}{l}\text { Assessment of Depression and Anxiety in Breast Cancer } \\
\text { Patiens: Prevalence and Associated Factors }\end{array}$ & $\begin{array}{l}\text { Konstantinos Tsaras } \\
\text { et al. }\end{array}$ & 2018 & Asia & 152 & Cohorte \\
\hline
\end{tabular}


En esta revisión se han incluido 6 estudios. La Tabla 1 presenta las principales características de los 6 estudios incluidos. Se aprecia que el periodo de publicación fue desde 2015 al 2018. Los estudios fueron desarrollados en personas de distintas etnias de
6 países que pertenecen a tres continentes diferentes, incluido Perú, Colombia, Irlanda, España y Malasia. Las muestras fueron muy heterogéneas, la población iba de 80 a 1086 pacientes.

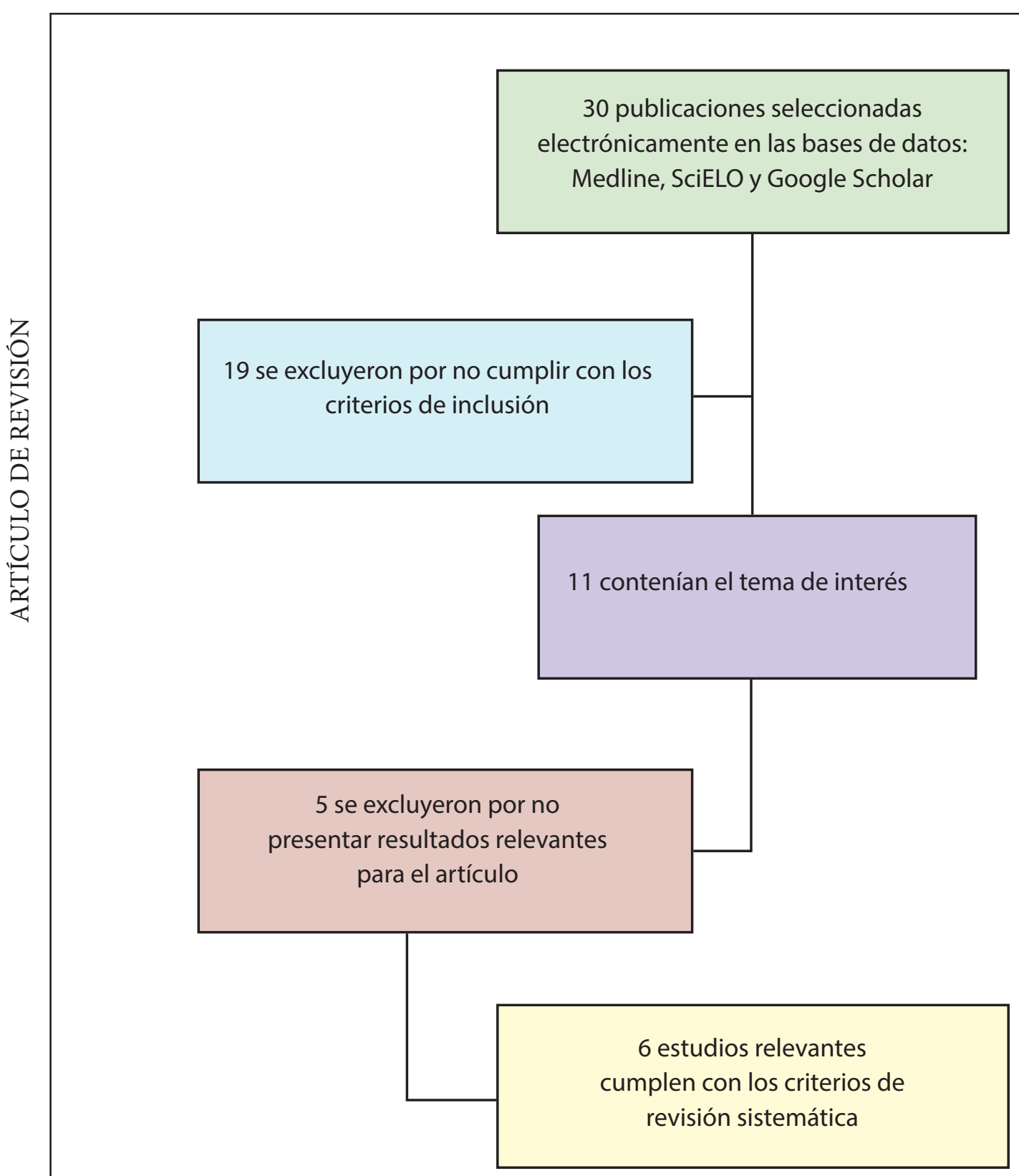

Figura 1. Identificación de los estudios y proceso de selección.

La tabla 2 muestra las características sociodemográficas asociadas a depresión en pacientes con cáncer de mama, estos incluyen la edad donde se ve que la población entre 40 y 60 años, estar divorciada o soltera está más asociado a sufrir depresión a comparación con los pacientes que están casadas.

En asociadas se encontró mayor asociación al grado de instrucción hasta primaria con depresión a comparación de los pacientes que tenían educación superior que fueron menos. Estar desempleado y pertenecer a una clase social baja aumenta el riesgo de tener depresión en pacientes con cáncer de mama.

En la tabla 3 se muestran los factores asociados a depresión en pacientes con cáncer de mama en relación a la terapia y estadío clínico. Donde los factores de riesgo son los pacientes en estadío clínico 4 y el tratamiento de quimioterapia a comparación de los pacientes sometidos a radioterapia que fue menor el número de pacientes con depresión. 
Tabla 2. Características sociodemográficas de los estudios incluidos en la revisión.

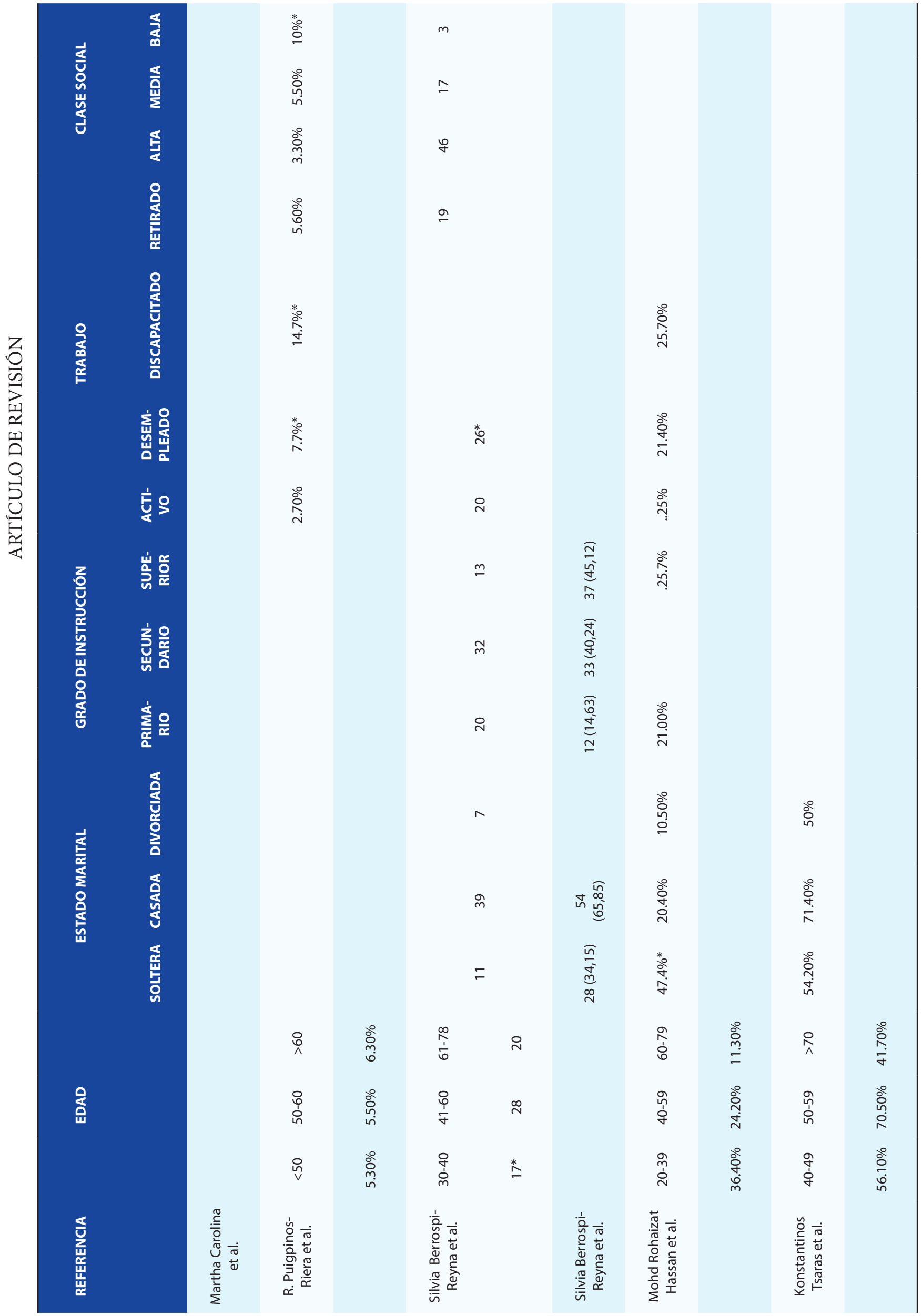


Tabla 3. Características de los estudios incluidos en la revisión según el estadio clínico y el tipo de tratamiento.

\begin{tabular}{|c|c|c|c|c|c|c|c|c|c|c|}
\hline \multirow[b]{2}{*}{ REFERENCIA } & \multicolumn{3}{|c|}{ TIPO DE TRATAMIENTO } & \multirow[b]{2}{*}{$\mathbf{I}$} & \multicolumn{5}{|c|}{ ESTADO CLÍNICO } & \multirow[b]{2}{*}{ IV } \\
\hline & $\begin{array}{l}\text { MASTEC- } \\
\text { TOMIA }\end{array}$ & $\begin{array}{l}\text { QUIMIO- } \\
\text { TERAPIA }\end{array}$ & $\begin{array}{l}\text { RADIOTE- } \\
\text { RAPIA }\end{array}$ & & IIA & IIB & IIIA & IIIB & IIIC & \\
\hline $\begin{array}{l}\text { Martha Carolina } \\
\text { et al. }\end{array}$ & & & & & & $\begin{array}{c}7,18 \pm \\
2,27\end{array}$ & $\begin{array}{c}8,84 \pm \\
1,54\end{array}$ & $\begin{array}{c}8,65 \pm \\
1,68\end{array}$ & $\begin{array}{c}8,60 \pm \\
0,97\end{array}$ & $\begin{array}{c}9,25 \pm \\
2,02\end{array}$ \\
\hline \multicolumn{11}{|l|}{$\begin{array}{l}\text { R. Puigpinós- } \\
\text { Rivera et al. }\end{array}$} \\
\hline \multicolumn{11}{|l|}{$\begin{array}{c}\text { Jovana Cvetkovic } \\
\text { et al. }\end{array}$} \\
\hline $\begin{array}{l}\text { Silvia Berrospi- } \\
\text { Reyna et al. }\end{array}$ & $190(62,71)$ & $221(72,94)$ & $\begin{array}{c}125 \\
(41,25)\end{array}$ & $\begin{array}{c}14 \\
(17,07)\end{array}$ & $\begin{array}{c}34 \\
(41,46)\end{array}$ & & $\begin{array}{c}90 \\
(29,70)\end{array}$ & & & $9(2,97)$ \\
\hline \multicolumn{11}{|l|}{$\begin{array}{l}\text { Mohd Rohaizat } \\
\text { Hassan et al. }\end{array}$} \\
\hline $\begin{array}{l}\text { Konstantinos } \\
\text { Tsaras et al. }\end{array}$ & $61.30 \%$ & $62.90 \%$ & $54.20 \%$ & $69.20 \%$ & $69.80 \%$ & & $61.20 \%$ & & & $31.3 \% *$ \\
\hline
\end{tabular}

\section{DISCUSIÓN}

Disponemos de pocos estudios que analizan los factores asociados a depresión en pacientes con cáncer de mama, especialmente en el Perú no existen estudios con poblacionales que aborden la problemática de depresión en pacientes con cáncer de mama, pese a que la salud mental ha sido la segunda prioridad de investigación en salud en el Perú6.

Uno de las principales complicaciones fueron la cantidad de factores asociados a depresión en pacientes con cáncer de mama, teniendo variaciones en cada artículo. Ya que muchos factores pueden intervenir en la génesis de síntomas depresivos en pacientes con cáncer de mama, se necesitan estudios adicionales a mayor escala para definir si existen factores adicionales asociados a la presencia de síntomas depresivos ${ }^{6}$.

Aunque algunos autores han planteado que en poblaciones similares las pacientes más jóvenes presentan mayor riesgo de depresión ${ }^{1}$ como en el estudio incluido hecho por Jovana Cvetković et al dice que hay mayor factor de riesgo en pacientes entre 30-40 años.

Estar divorciada o soltera está más asociado a sufrir depresión a comparación con los pacientes que están casadas, en el artículo hecho por Mohad Rohaizat Hasssan et al. Se observa que el mayor porcentaje de pacientes con depresión y cáncer de mama son solteras y están entre la edad de 20 y 40 años?.
En el estudio hecho por Konstantinos Tsaras et al. Se observó que el $50 \%(p<0.05)$ de las pacientes divorciadas sufrían de depresión y más del $70 \%$ estaba entre la edad de 50 y 59 años.

En el estado de educación en la revisión se encontró que las personas con grado de educación hasta primaria era un factor de riesgo, comparado con un estudio fue que las mujeres menos educadas que lidian con el cáncer de mama tenían más probabilidades de experimentar depresión según informaron hallazgos similares.

De acuerdo con nuestros resultados, los pacientes con nivel socioeconómico tienen mayor probabilidad de experimentar síntomas depresivos que confirman estudios previos ${ }^{11}$.

Respecto a la proporción de pacientes con sintomatología depresiva entre aquellas sometidas a quimioterapia, radioterapia y mastectomía radical, se encontró mayor riesgo en pacientes sometidas a quimioterapia

Finalmente, de acuerdo con nuestros resultados, los pacientes que fueron diagnosticados en estadio IV de cáncer de mama tenían un alto riesgo de depresión en comparación con los pacientes en estadio I.

Este hallazgo está de acuerdo con un estudio reciente entre cáncer de mama en Grecia (Fradelos et al., 2017) en el que los pacientes con cáncer de mama en estadio IV tenían 1.9 ( $p=0.003$ ) más probabilidades de experimentar síntomas depresivos. 


\section{CONCLUSIÓN}

Se concluye en la presente revisión que hace falta más estudios a mayor escala respecto a los factores asociados a depresión en pacientes con cáncer de mama especialmente a nivel de Perú, ya que se encontró solo un artículo relacionado al tema de interés.

Por otra parte hace falta sistematizar bien los factores asociados ya que se encuentran diversos factores relacionados a depresión y con diferentes resultados dependiendo del tipo de hospital y de país, para poder plantear un buen programa de apoyo psicológico y educación sobre la enfermedad.
Contribuciones de autoría: Los autores participaron en la concepción, recolección de información, redacción y aprobación de la versión final del artículo.

Financiamiento: Autofinanciado.

Conflicto de interés: Los autores declaran no tener conflicto de interés en la publicación de este artículo.

Recibido: 19 de noviembre del 2018

Aprobado: 20 de diciembre del 2018

Correspondencia: Ximena Vanessa Arbulú La Torre

Dirección: Pasaje Eliot Estela Mz. E-3 Lote 16 Urb. Honor y Lealtad. Santiago de surco Celular: +51981271994

Correo:ximena.0694@hotmail.com

\section{REFERENCIAS BIBLIOGRÁFICAS}

1. Valderrama Rios, M. C., \& Sánchez Pedraza, R. Trastornos de ansiedad y depresión en relación con la calidad de vida de pacientes con cáncer de mama en estadio localmente avanzado o diseminado. Rev Col Psiquiat. 2018; 47(4):211-220.

2. Lic. Educ. Esp. Biolog. Elvira Ramona Peña Guerra, I Lic. Cult. Fis. Dep. Rec. Aliev Fonseca Peña,II Esp. 1er Grado MGI Nilda Meireles Aldana. Anxiety and depression in women with breast cancer: Social and clinical determinants and influence of the social network and social support. Cancer Epidemiology 55. 2018; 123-129

3. Lic. Educ. Esp. Biolog. Elvira Ramona Peña Guerra, I Lic. Cult. Fis. Dep. Rec. Aliev Fonseca Peña,ll Esp. 1er Grado MGI Nilda Meireles Aldana .Evaluación y control de los estados emocionales en pacientes con cáncer de mama. Multimed 2013; 16(3)

4. Sheila Pintado. Análisis predictivo del bienestar emocional en pacientes con cáncer de mama. Psicología y Salud. (2017). 2017; 27(2):223-229.

5. JovanaCvetković $\mathrm{n}$, MilutinNenadović. Depression in breast cancer patients. Psychiatry Research 240. 2016; 347-347.
6. Sheila Pintado. Información médica sobre el cáncer de mama y repercusiones psicológicas en pacientes oncológicos provenientes de España durante el año 2011. MÉD.UIS. 2017;30 (3):13-20.

7. Berrospi-Reyna, S, Herencia-Souza M, Soto A. Prevalencia y factores asociados a la sintomatología depresiva en mujeres con cáncer de mama en un hospital público de Lima, Perú. Acta Med Peru. 2017;34 (2):95-100.

8. Sheila Pintado. Self-concept and emotional well-being in patients with breast cancer.Revista Iberoamericana de Psicología y Salud. 2017; 8(2):76-84.

9. Hassan, M. R., Shah, S. A., Ghazi, H. F., Mohd Mujar, N. M., Samsuri, M. F., \& Baharom, N. Anxiety and depression among breast cancer patients in an urban setting in Malaysia. Asian Pac J Cancer Prev. 2015; 16(9): 4031-5. 10. Tsaras, K., Papathanasiou, I. V., Mitsi, D., Veneti, A., Kelesi, M., Zyga, S., \& Fradelos, E. C. Assessment of Depression and Anxiety in Breast Cancer Patients: Prevalence and Associated Factors. Asian Pacific journal of cancer prevention. 2018;19(6):1661.

\section{Indizado en: latindex}

http://www.latindex.org/latindex/ficha?folio $=14280$

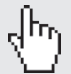

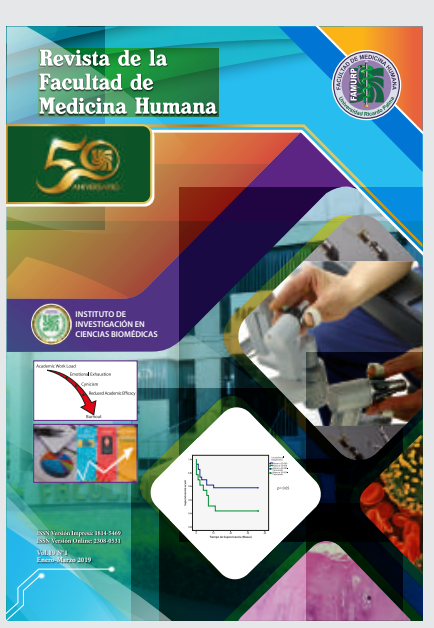

